\title{
Género y encierro, sentidos en tensión
}

\section{Gender and imprisonment, the tension in between}

\author{
Verónica Loyola \\ veronicaloyola17@gmail.com \\ Unidad 40 de Lomas de Zamora, Servicio Penitenciario \\ Bonaerense, Argentina \\ Marisa Teplisky \\ marisateplisky@gmail.com \\ Unidad 40 de Lomas de Zamora, Servicio Penitenciario \\ Bonaerense, Argentina
}

Recepción: 30 Marzo 2019

Aprobación: 14 Julio 2020

Publicación: 01 Marzo 2021

Cita sugerida: Loyola, V. y Teplisky, M. (2021). Género y

encierro, sentidos en tensión. Descentrada, 5(1), e139.

https://doi.org/10.24215/25457284e139

\begin{abstract}
Resumen: En este artículo nos interesa compartir reflexiones en torno a una experiencia de trabajo llevada a cabo durante el año 2018 en el marco de un taller socioeducativo sobre género y sexualidades. En el mismo, participaron un grupo de mujeres detenidas en el anexo de la Unidad Penal $N^{\circ} 40$ de Lomas de Zamora, perteneciente a la órbita del Servicio Penitenciario Bonaerense. Nos proponemos analizar los alcances y limitaciones de dicha experiencia, así como también socializar las vivencias atravesadas como talleristas/coordinadoras. Asimismo, arribar a conclusiones que propicien la posibilidad de capitalizar errores y aciertos en el abordaje de los encuentros en pos de ampliar y fortalecer espacios tratamentales con perspectiva de género en contextos de encierro.
\end{abstract}

Palabras clave: Mujeres encarceladas, Contexto de encierro, Tratamiento penitenciario, Perspectiva de género.

Abstract: In this article we are interested in sharing reflections on a work experience carried out during 2018 within the framework of a socio-educational workshop on gender and sexualities that was attended by a group of women inmates from the Criminal Unit No. 40 from Lomas de Zamora, Buenos Aires Penitentiary Service. We intend to analyze the scope and limitations of this experience as well as to share our experience as workshop coordinators, hopping to arrive to conclusions that will clarify the mistakes, and achievements, that were made while organizing the meetings in order to provide a guideline for other coordinators or penitentiary staff so that they will be able to carry out similar workshops or other prision programs with a gender perspective in confinement contexts.

Keywords: Women inmates, Jail, Gender Perspective, Gender oriented prision programs.

\section{INTRODUCCIÓN}

En este trabajo compartiremos reflexiones en torno a una experiencia de intervención socioeducativa en contexto de encierro, que se llevó cabo en el año 2018 a través de un dispositivo de taller denominado "Género y Sexualidades". Este dispositivo se enmarcó en lo que se denomina "tratamiento penitenciario" y fue llevado a cabo en el anexo femenino de la Unidad penal $N^{\circ} 40$ de Lomas de Zamora, dependiente del Servicio Penitenciario Bonaerense (SPB). Cabe destacar que en la provincia de Buenos Aires existen 57 cárceles, de las cuales tan solo 4 están destinadas a albergar exclusivamente a mujeres. La Unidad 40 de Lomas de Zamora 
se encuentra ubicada en lo que se denomina "conurbano bonaerense", que es el área que rodea a la ciudad Autónoma de Buenos Aires.

A lo largo del trabajo denominamos "tratamiento penitenciario" a todos aquellos dispositivos o estrategias de intervención en torno a distintos aspectos, tales como educación, salud psicofísica, trabajo, deporte y recreación, entre otros, que se ofrecen desde el SPB y/o desde la sociedad civil a las personas detenidas. Estos tienen por objeto promover la adquisición de recursos simbólicos e intelectuales, así como desarrollar capacidades prácticas que puedan ser capitalizadas al momento de la reinserción social de lxs detenidxs.

De nuestra trayectoria profesional ${ }^{1}$ de trece años de trabajo en el ámbito del Servicio Penitenciario Bonaerense, observamos que el tratamiento penitenciario responde a la cultura androcéntrica en la cual las necesidades particulares de las mujeres detenidas no son contempladas en su especificidad. Esto lo advertimos, en el ejercicio de nuestra práctica profesional, en la que desarrollamos nuestra tarea como psicóloga (en el área sociolaboral) y socióloga (en el área social) en el marco de un equipo técnico interdisciplinario. Se destaca que en todas las unidades de SPB funciona un Departamento Técnico Criminológico (DTC), cuyo principal objetivo es responder a oficios judiciales a partir de informes confeccionados por las distintas áreas de tratamiento penitenciario, por ejemplo: área educativa, sanidad, asistencia social y sociolaboral, entre otras. A partir de este encuadre institucional formal en el cual nos desarrollamos, ambas hemos detectado, en las entrevistas realizadas a mujeres, un conjunto de necesidades y demandas insatisfechas cuyos emergentes comunes eran la pérdida de contacto filiatorio, la imposibilidad de acceder a información sobre el estado de sus causas, limitaciones en el acceso a la salud sexual y reproductiva, abandono por parte de sus parejas a raíz de su detención y ausencia de acompañamiento familiar.

A partir del intercambio de esta información nos planteamos generar un dispositivo tratamental alternativo a la oferta institucional dada, en el cual poder abordar algunas de estas problemáticas desde un enfoque de género.

Para ello en una primera etapa, durante el año 2017, iniciamos un proceso de entrevistas a las mujeres detenidas. La metodología utilizada fue la etnografía de campo. Dicha elección se debió a la posibilidad de ahondar en los sentires y vivencias que permitieran recuperar y sistematizar información sobre las necesidades de las mujeres. El material obtenido de las entrevistas nos permitió gestionar un dispositivo de intervención específico para mujeres en formato taller. En este sentido, los ejes temáticos del mismo se circunscribieron a aspectos vinculados al ejercicio de la maternidad, el sostenimiento del vínculo con sus hijxs y el cuidado de lxs mismxs, como así también las relaciones sexo-afectivas y cuestiones asociadas al campo de la salud sexual y reproductiva.

\section{LA CÁRCEL FUE Y ES PENSADA PARA VARONES}

Las mujeres son diferentes, y viven su encarcelamiento de forma diferente y por ello hay que promover para ellas medidas especificas (Yagüe Olmos, 2007, p. 7).

La Ley de Ejecución Penal 12.256 es la normativa que regula el funcionamiento del Servicio Penitenciario Bonaerense. El artículo 4 detalla que "la adecuada inserción social de los procesados y condenados a través de la asistencia o tratamiento y control” es su principal función. Para ello, la cárcel como institución destinada a ejecutar la pena, debe ofrecer un aspecto tratamental, es decir, debe implementar estrategias de intervención de índole laboral, socio educativo, sanitario, psicoasistencial, recreativo, etc., que permitan capitalizar productivamente el tiempo de detención de lxs detenidxs y a la vez activar procesos de resignificación en torno al posicionamiento subjetivo frente al delito, con miras a facilitar el proceso de reinserción social.

La Argentina suscribe a normativa internacional que recomienda a los Estados parte, desde una perspectiva de Derechos Humanos, diversas formas de atenuar el impacto negativo del encierro en las personas. En esta línea, las Reglas de Bangkok sostienen que lxs varones y mujeres detenidxs no deben recibir un "trato 
igual", sino, por el contrario, debe asegurarse un trato diferente, bajo leyes y políticas sensibles al género de las personas.

De nuestra trayectoria profesional en contextos de encierro, advertimos que estas recomendaciones no se cumplen en la práctica institucional. El tratamiento penitenciario indiferenciado produce vulneración de derechos y arroja a las mujeres a una "doble condena": social y penal.

El género se conceptualizó como el conjunto de ideas, representaciones, prácticas y prescripciones sociales que una cultura desarrolla desde la diferencia anatómica entre mujeres y hombres, para simbolizar y construir socialmente lo que es "propio" de los hombres (lo masculino) y "propio" de las mujeres (lo femenino)" (Lamas, 2000, p. 2).

Un fenómeno reciente ha sido el crecimiento sostenido del encarcelamiento de mujeres. El informe anual de $2015,{ }^{2}$ producido por el Sistema Nacional de Estadística sobre Ejecución de la Pena (SNEEP), registra que en la provincia de Buenos Aires había 1148 mujeres detenidas, incrementándose hacia el $2017^{3}$ en un total de 1326. Sin embargo, la cárcel como institución androcéntrica, hace caso omiso en términos de género a las transformaciones que ha sufrido en la composición y en las particularidades de su población.

\section{GÉNERO Y DELITO: "LA DOBLE CONDENA"}

Las mujeres reclusas cumplen el doble castigo (social y penal) de la prisión y de la sociedad (...) las personas están en prisión como castigo, no para recibir castigos. En este sentido, las circunstancias del encarcelamiento no deberían utilizarse como castigo adicional, debiendo así reducirse en la medida de las posibilidades los efectos negativos de la prisionalización (Rodríguez, 2005, p. 4).

La desigualdad de género como problemática social incide en la prisionalización de las mujeres. En este sentido, las mujeres que se encuentran detenidas en las unidades penitenciarias de la provincia de Buenos Aires han atravesado vivencias compartidas que están vinculadas a experiencias de discriminación, violencia de género, maltrato, exclusión y falta de acceso a los derechos básicos, como educación, vivienda, salud y trabajo. "Estas tramas de violencias previas al encierro son aspectos nodales para dar entidad política e histórica a las experiencias de las mujeres detenidas" (Colanzi, 2015, p. 4). Muchas son las investigaciones (CELS, 2011; Pegoraro, 2010) que evidencian cómo las condiciones de pobreza y extrema vulnerabilidad en la que las mujeres viven previo a su detención inciden en la incursión de éstas en el accionar trasgresor.

La mujer en situación de encierro ha sido históricamente olvidada e invisibilizada como población con necesidades y características particulares. Como ya se señaló, de un total de 57 Unidades Penitenciarias dentro del SPB, sólo 4 están destinadas exclusivamente a población femenina. En paralelo coexisten cárceles mixtas, que fueron diseñadas para alojar a varones y que luego han sido, por una cuestión de necesidad, utilizadas para alojar a población femenina en un anexo. Este es el caso de la Unidad 40 de Lomas de Zamora, donde llevamos a cabo el taller al que haremos referencia en las próximas líneas.

En función de los datos recabados a lo largo de las entrevistas y de nuestra experiencia en este contexto, se desprende que el hecho de que la oferta tratamental sea la misma para varones y mujeres, sella y profundiza la desigualdad de género. Esto da cuenta de que en el cuerpo de la mujer detenida se entretejen mecanismos de sometimiento e invisibilización, producto tanto de la inscripción de una cultura carcelaria, pero además de la inscripción en un sistema patriarcal que imprime sus sesgos, dentro y fuera de los muros (Colanzi, 2015, p.10).

Respecto a lo institucional, muchas son las áreas tratamentales en las que advertimos el sesgo androcéntrico del dispositivo carcelario y observamos los efectos del mismo en lxs cuerpos y mentes de las mujeres que lo padecen. En el plano de la salud vemos que, en la provincia de Buenos Aires, la administración del servicio encuentra a cargo del área de salud penitenciaria del SPB, en lugar del Ministerio de Salud. En la práctica, la falta de articulación de estas entidades segrega a las mujeres del acceso a las campañas de salud y políticas nacionales y provinciales que promocionan los derechos sexuales y reproductivos de las mujeres. En este 
marco, la falta de dispositivos específicos del género, la ausencia de equipamiento y profesionales ginecólogxs idóneos para realizar controles periódicos, han sido relevados como una de las mayores preocupaciones de las mujeres detenidas a lo largo de las entrevistas. Gladys ${ }^{4}$ comentaba: "Me pasó que estuve 15 días con pérdidas y cuando me desmayé me sacaron al hospital porque no hay ginecólogxs”.

En el plano de la salud mental y emocional, las mujeres por su condición de género, vivencian las relaciones vinculares, fundamentalmente las materno-filiales, en estos contextos con un mayor nivel de malestar psíquico. El distanciamiento de los estereotipos de género, asociados generalmente a la maternidad, en torno a lo que "debe ser" y "debe hacer una mujer" ocasiona mayor ansiedad, impactando en su estado de salud por el mayor sufrimiento psicológico que implica. El impacto que en ellas tiene el encierro en ese sentido es invisibilizado, en tanto que no se brinda tratamiento específico.

En esta línea, al pensar en el deterioro de las condiciones psicofísicas y emocionales que sufre una mujer al caer detenida, se le suma la discontinuidad en el sostenimiento de las visitas. En este sentido, a diferencia de los varones, quienes son visitados regularmente por sus parejas, las mujeres no gozan de esa frecuencia en las visitas y, en ocasiones, no cuentan con visita alguna, a pesar de haber estado en relación de pareja hasta el momento previo a su detención. Esto surge de datos obrantes en el área de visitas de la dependencia y de conversaciones en el marco del taller: "Desde que caí detenida mi pareja me dejó tirada, pero ya sabía porque él trabaja y se le complica”. Los dichos denotan la naturalización de estas conductas, aunque esto no evita que las mismas sean vivenciadas con menos angustia y dolor durante el proceso de detención.

Las relaciones sexo-afectivas se vivencian en el marco de "visitas íntimas". Estas se llevan a cabo en espacios cerrados destinados al encuentro íntimo con una pareja sexual. En el marco de conversaciones, las participantes del taller refieren que el espacio destinado a tal fin no cumple con los requisitos básicos de limpieza, tampoco son provistxs de elementos de higiene y profilaxis. Una de las consecuencias de estas deficiencias suele ser el afrontamiento de embarazos no deseados y enfermedades de transmisión sexual, que luego no son advertidas o controladas como corresponde. "Las mujeres son violentadas, desde las prácticas penitenciarias, de un modo que apunta a su mundo más íntimo y doméstico: el de la limpieza, el cuidado del cuerpo y el temor a los contagios y la enfermedad" (Daroqui, Fridman y Maggio, 2006, p. 93).

Las demandas de las mujeres detenidas hacia el personal de seguridad giran en torno a cuestiones sociovinculares (averiguar dónde están sus hijxs, poder comunicarse con ellxs, visitas, etc.) y demandas de atención de su salud mental y física. De los relatos surge que eventualmente estas no son consideradas válidas y se desestiman o minimizan. Asimismo, el criterio para viabilizar las demandas referidas varía en función de quien las reciba, aumentando esto los niveles de ansiedad y malestar.

Las formas en que se expresa el poder en las prácticas cotidianas pueden conducir a dos situaciones contrapuestas: si se aplica de acuerdo a lo que manda la ley y los reglamentos o si se aplica en forma discrecional, de acuerdo a los intereses coyunturales tanto de internos como de agentes penitenciarios. De la primera forma, la percepción por parte de los detenidos será la de un poder coercitivo pero que alcanza a todos por igual de manera que se estaría aplicando con criterios de razonabilidad. La segunda situación es la que establece diferencias entre los internos, con un trato discrecional y que no puede preverse (Kalinsky, 2008, p. 49).

Esto da cuenta de que, pesar de que en la Argentina esté vigente la Ley Micaela, ${ }^{5}$ la ausencia de formación con perspectiva de género del personal penitenciario produce un efecto adverso que refuerza la violencia institucional.

Otro factor de desigualdad es el funcionamiento de una justicia patriarcal que criminaliza el narcomenudeo, es decir, que castiga especialmente al eslabón más débil de la cadena del narcotráfico, mayormente ocupado por mujeres. En este sentido, podemos destacar que las mujeres que viven en estos contextos en formas más marcada que los hombres, "son jóvenes sin un empleo previo, que no tienen antecedentes penales, que están detenidas sin una condena definitiva y que están imputadas fundamentalmente por infracción a la ley drogas" (Olaeta, 2011, p. 113). En el marco de conversaciones informales, advertimos que este tipo de delito las habilita a dar continuidad a su rol de cuidadora a la vez que 
proveedoras económicas. Los dichos de Estela refuerzan lo señalado anteriormente: "Era esto o prostituirme y no podía dejar solxs a mis hijxs. Tengo siete, imaginate".

La desigualdad de género opera de este modo promoviendo un desproporcionado encarcelamiento impulsado desde la lógica patriarcal. La escalada en el aumento de mujeres detenidas no se debe al aumento del número o la gravedad de los delitos cometidos, sino más bien a un cambio en los criterios de los tribunales sentenciadores y en las prioridades de las políticas de orden público. Tiene que ver con la criminalización del consumo y el tráfico de drogas (CELS, 2011, p. 11).

El recorrido hecho hasta el momento tiene la intención de explicitar los motivos por los cuales se torna indispensable la implementación de propuestas tratamentales desde una perspectiva de género en las cárceles.

La perspectiva de género permite analizar y comprender las características que definen a las mujeres y a los hombres de manera específica, así como sus semejanzas y diferencias. Esta perspectiva de género analiza las posibilidades vitales de las mujeres y los hombres: el sentido de sus vidas, sus expectativas y oportunidades, las complejas y diversas relaciones sociales que se dan entre ambos géneros, así como los conflictos institucionales y cotidianos que deben enfrentar y las maneras en que lo hacen (Lagarde, 1996, p. 2).

Trabajar con este enfoque significa asumir una postura política a favor de la igualdad y equidad para la construcción de una sociedad más igualitaria y humana, donde las diferencias entre hombres y mujeres no sean factor de discriminación, opresión y explotación.

\section{ACERCA DE LA EXPERIENCIA DEL TALLER}

Las mujeres infractoras eran, pues, por rebelarse al rol social-sexual asignado, ¡las pecadoras sexuales, las brujas, las ebrias, las de vida desordenada, las desobedientes! Nada ha cambiado demasiado, como veremos, a pesar del grado de incorporación de la mujer al trabajo productivo, sólo que ahora lo vemos más sutilmente reflejado (Aniyar de Castro, 2002, p. 338).

Para la conformación del grupo de participantes del taller, se nos presentó un primer obstáculo. La propuesta original, que fue rechazada, era incorporar a mujeres de los distintos pabellones con el fin de generar un espacio inclusivo y desmitificar la idea de que mujeres de distintos pabellones no pueden compartir espacios comunes por ser “conflictivas”. Finalmente, se realizó con las mujeres alojadas en el pabellón 4, el más estigmatizado por las autoridades, dado el alto nivel de conflictividad. Se destaca la exclusión del mismo de actividades tratamentales en general. "Estamos engomadas todo el día", decía Nataly, interna del pabellón 4.

Del taller participaron 15 mujeres, cuyas edades oscilaban entre los 20 y 50 años, con diversidad en términos de identidad de género y orientación sexual. Sólo una de ellas no era madre, el resto tiene a sus hijxs con familiares directos o allegadxs. Ninguna convive con sus hijxs, dado que este anexo no cuenta con una infraestructura adecuada conforme lo estipula la ley. ${ }^{6}$ La duración del taller fue de seis meses con encuentros semanales de una hora y media, y se realizó en la escuela intramuros de la dependencia. ${ }^{7}$

El propósito del taller fue habilitar un espacio de escucha, encuentro y socialización de experiencias acerca de las formas en las que se vivencian los vínculos afectivos y las tensiones inherentes a la situación de detención, con la idea de afianzar las fortalezas de las mujeres en este campo.

Los debates en torno a lxs feminismos y el surgimiento del colectivo "Ni una Menos", 8 permitieron establecer un marco en el cual dar sentido al ofrecimiento del espacio de reflexión en torno a géneros y sexualidades.

En el primer encuentro, bajo la consigna "acordar normas de convivencia", las chicas establecieron las siguientes: "no juzgarnos entre nosotras", "no contar en el pabellón lo que decimos acá", "escucharnos y respetarnos", entre otras. Todo lo escrito versaba en torno a la confianza y la confidencialidad, ambos aspectos valorados ampliamente en este contexto. Sentimos a lo largo de toda la experiencia que estas normas fueron los soportes simbólicos que favorecieron que el taller se encuadre en un marco de respeto, escucha amorosa y 
cuidado. Así lo expresó Anahí, en un encuentro posterior:"Lo que me gusta es que en el pabellón nadie habla de lo que contamos en el taller, muere ahí".

En un comienzo trabajamos en torno al eje socialización y género. En este sentido, a través de actividades lúdicas, propusimos compartir vivencias, sensaciones en relación a las infancias. Aquí apareció mayoritariamente la naturalización de roles de género estereotipados, como el cuidado de hermanxs menores, la asunción temprana de responsabilidades de adultas (trabajo infantil) y la ausencia de figuras adultas masculinas referentes durante sus primeras infancias.

En los sucesivos encuentros profundizamos en estas temáticas con material audiovisual (publicidades sexistas) para abordar la cuestión de los estereotipos de género y violencia simbólica en los medios de comunicación. Al indagar acerca de las sensaciones y percepciones luego del visionado, lo que afloró fue cierta distancia respecto a las representaciones estereotipadas de las mujeres que aparecían en las publicidades. "Yo ni tengo para comer menos para comprar Mr. musculo", otras hacían chistes o ponían cara de desconcierto. $\mathrm{Al}$ respecto, entendimos que el recurso fue elegido desde una visión sociocentrista, debiendo reformularse. No ocurrió lo mismo con un compendio de canciones sexistas que estaban más cerca de su universo simbólico y cultural, lo cual permitió trabajar en torno a los conceptos de violencia simbólica como un tipo de violencia de género que se encuadra bajo esta modalidad mediática.

Para abordar la violencia de género, optamos por trabajar con los mitos del amor romántico. ${ }^{9}$ Nos propusimos generar una posición crítica respecto a estos con el objetivo de desnaturalizar vínculos violentos. Para ello utilizamos como recurso viñetas ${ }^{10}$ que los graficaban. La consigna era identificarlos y contrastarlos con sus propias vivencias. Este disparador provocó verborragia y necesidad de expresar y comunicar sus propias experiencias. A parecían relatos en donde habían "dejado todo por amor". Carla contaba: "a los quince cuando quedé embarazada me escapé de mi casa en Paraguay y lo vine a buscar a Buenos Aires, sólo tenía la dirección". Así surgieron historias de amor en la cárcel, algunas de ellas con contenido violento, embarazos transitados en soledad y en contextos de extrema vulnerabilidad. Espontáneamente relataban episodios de violencia de género y maltrato ejercido por sus parejas: "mi límite fue cuando me apuntó en la cabeza y me gatilló con su arma, estoy viva de milagro", "me sentí libre cuando lo mató la policía”, "cada vez que quedaba embarazada me golpeaba la panza”. Fue un denominador común evocar episodios de violencia extrema en sus vínculos de pareja. Cinco de las quince mujeres relataron el mismo evento de violencia ejercida mediante un arma de fuego.

Luego de compartir esas historias quedamos conmovidas. Continuamos con un ejercicio de autoconocimiento en el cual pudieran visualizar o rememorar una escena agradable en la que se hayan sentido queridas y respetadas. Sorprendió que muchas aludieran recuerdos asociados a sus hijxs y mascotas. Esto favoreció el trabajo reflexivo en torno a revalorizar el amor bajo otros formatos distintos al del amor romántico.

En el encuentro siguiente retomamos los relatos de la práctica anterior para introducir la problemática de la violencia de género como problema social. Uno de los objetivos de este encuentro estaba dirigido a reflexionar sobre cómo las vivencias personales tienen un carácter político y social. Las intervenciones y preguntas que surgieron nos permiten encuadrar la temática del movimiento "Ni una menos" y contextualizarla en el marco de una actividad en la cual dispusimos un círculo de bancos, en cuyo respaldo se presentaba una breve biografía de una mujer víctima de femicidio. ${ }^{11}$ Surgió de forma espontánea el interés por participar simbólicamente en la marcha anual de "Ni una menos" a través de la confección de un cartel. Colectivamente, produjeron el escrito: "El feminismo nunca ha matado a nadie. El machismo mata cada día!. Mujeres detenidas presentes! Unidad $N^{\circ} 40$ Pabellón 4". Llevamos el mismo al encuentro y, posteriormente, socializamos la experiencia a través de registros audiovisuales.

La segunda parte del taller se centró en el trabajo sobre salud sexual y reproductiva, y maternidad. Los encuentros buscaron "poner en cuestión sus saberes en relación a su cuerpo, su sexualidad y sus prácticas de cuidado” (Comas y Otero, 2014, p. 9). Apelamos a la construcción colectiva del conocimiento. 
El uso de un recurso lúdico "pescando ideas falsas en torno a la anticoncepción" 12 funcionó para que las chicas pudieran expresar dudas sin verse expuestas o sentirse juzgadas por sus elecciones y formas de vivir su sexualidad. No obstante, como varias de las mujeres practican el culto evangélico, algunas de las respuestas en torno a identidades de género no binaries, daban lugar a cuestionamientos. En momentos de tensión, un recurso que facilitó la dinámica fue invocar a las normas de convivencia establecidas al comienzo, "escucharse y no juzgar a la compañera”.

La temática del aborto devino a raíz de un intercambio en torno a la maternidad y surgió espontáneamente luego de que Gabriela exprese “de los 10 hijxs que tuve, la verdad es que sólo desee a unx”. Muchas se oponían a esta idea refiriendo "el aborto es de infanto". La figura de la "infanto" es un término de la jerga carcelaria que refiere a la mujer que asesina a su propio hijo (infanticidio). Es una figura demonizada dentro del ámbito carcelario. Por este motivo, muchas se reprimen de manifestarse a favor o de hablar al respecto del derecho al aborto. Se suma a esto que la mayoría adhiere a concepciones religiosas y acompañan sus argumentos. No obstante, se dio lugar a conversar sobre los derechos sexuales y reproductivos. Muchas se refirieron a las visitas íntimas: "cuando llegas ya fue, estás ahí y no hay preservativos", "me pasó de querer cuidarme y no poder". Esta dinámica habilitó el abordaje de los distintos tipos de violencia de género y los ámbitos en los que se manifiestan. ${ }^{13}$

Por último, compartimos las vivencias de uno de los últimos encuentros en los que se trabajó sobre maternidad con el objetivo de cuestionar los estereotipos de género en torno a este rol y la desidealización del mismo.

Cuando las mujeres presas verbalizan su ideal de madre, este modelo responde a un rol totalmente tradicional y estereotipado de madre como la cuidadora por excelencia (...) es especialmente sorprendente cuando, además, muchas veces se trata de mujeres que en sus trayectorias vitales han caracterizado por estar alejadas de ese ideal de mujer (Igareda, 2006, p. 62).

Por medio de un trabajo de escritura, cada mujer pudo expresar lo que le gusta de ser madre. Nos propusimos reforzar el potencial de cada una y fortalecer las subjetividades, pese a su condición de detención y las ausencias que eso conlleva.

\section{REFLEXIONES FinALES}

Las mujeres son un "anexo" (el anexo femenino) como se describe al espacio físico que ocupan en la unidad.

De la experiencia destacamos la posibilidad de ofrecer un espacio pensado por y para las mujeres, donde las mismas se sientan validadas en sus formas de sentir, pensar y actuar. Asimismo, consideramos que el taller fue condición de posibilidad para que las participantes pudieran creer y confiar en sus pares, dejando de lado, al menos en ese espacio, la suspicacia y la desconfianza inherentes a las dinámicas vinculares intracarcelarias. Se buscó en todo momento quebrar los circuitos de exclusión y habilitar un espacio en el que predomine la circulación de la palabra como alternativa, frente al endurecimiento de las condiciones propias del encierro.

A partir de la experiencia, se gestaron nuevos proyectos, surgidos del interés genuino de las participantes. En este sentido, se logró gestionar la apertura de una biblioteca para uso exclusivo del anexo femenino, la cual fue acondicionada por las mujeres del pabellón 4 como nuevo espacio tratamental. El taller fue advertido por las autoridades del penal como un antecedente positivo que influyó directamente en la disminución de los niveles de conflictividad entre internas. Esto da cuenta de una situación paradojal en el funcionamiento del sistema punitivo, la premisa del encierro como castigo cae frente a la posibilidad de brindar instancias de socialización y apertura a las necesidades específicas de las mujeres.

De nuestro paso por esta experiencia como talleristas nos llevamos, en principio, mucho afecto, la posibilidad de conocer historias de lucha de mujeres que han llegado a la cárcel luego de transitar lo inimaginable. Comprendimos también que estos espacios favorecen la capitalización del tiempo de detención desde una perspectiva de crecimiento personal y, a la vez, operan como puente hacia el fortalecimiento de las 
relaciones entre las mujeres, disminuyendo el nivel de conflicto y dificultades que, en su mayoría, provienen de la atomización y la individualidad, lógicas que imperan en los dispositivos de encierro.

Por lo antes referido, entendemos que la ausencia de propuestas tratamentales con perspectiva de género y la falta de formación del personal penitenciario en este enfoque, es parte del entramado patriarcal que golpea a lxs más débiles con más fuerza.

Las mujeres encarceladas deben ser tratadas en tanto sujetas de derechos. En este sentido, la necesidad de visibilizar esta problemática y generar políticas públicas con perspectiva de género que alcancen al ámbito carcelario debe asumirse como algo de carácter urgente. Por lo referido, entendemos que los encuentros propuestos se plantean como acciones contrahegemónicas, contraculturales, antipatriarcales y, por ello, imprescindibles y necesarias.

\section{Agradecimientos:}

Agradecemos especialmente a nuestrxs queridxs compañerxs: Paula, Noelia y Juan Pablo quienes nos acompañaron en esta práctica, enriqueciéndonos con sus aportes. Y a las participantes del taller que confiaron en nuestra labor y se comprometieron con esta experiencia.

\section{REFERENCIAS}

Aniyar De Castro, L. (2002). Las mujeres infractoras. Impacto y amplificación de los efectos de la pena. Capitulo Criminológico, 30(4), 333-351.

Centro de Estudios Legales y Sociales (CELS), Ministerio Público de Defensa (DGN) y Procuración Penitenciaria de la Nación (PPN) (2011). Mujeres en Prisión: los alcances del castigo. Buenos Aires: Siglo XXI. Recuperado de ht tps://www.cels.org.ar/web/wp-content/uploads/2011/04/Mujeres-en-prision.pdf

Comas, Alicia y Otero, Maria Eugenia (2014) Experiencias para armar. Manual para talleres en salud sexual y reproductiva. Ministerio de Salud de la Nación.

Colanzi, I. (2015). Los varones con las penas y las mujeres con los chicos. Concepciones que sustentan las prácticas frente a las mujeres y el contexto de encierro. Trabajo presentado en XI Jornadas de Sociología. Facultad de Ciencias Sociales, Universidad de Buenos Aires, Buenos Aires. Recuperado de: https://www.academia.edu/13846323/Los_varones_con_las_penas_y_las_mujeres_con_los_chicos._Con cepciones_que_sustentan_las_prácticas_frente_a_las_mujeres

Daroqui, A., Fridman, D. y Maggio, N. (2006). Voces del encierro: mujeres y jóvenes encarceladas en la Argentina. Una investigación socio-jurídica, Buenos Aires: Ediciones Jurídicas.

Argentina. Ministerio de Justicia, Seguridad y Derechos Humanos, Sistema Nacional de Estadísticas sobre Ejecución de la Pena (SNEEP) (2015) Informe Anual Buenos Aires SNEEP.

Argentina. Ministerio de Justicia, Seguridad y Derechos Humanos, Sistema Nacional de Estadísticas sobre Ejecución de la Pena (SNEEP) (2017) Informe Anual Buenos Aires SNEEP.

Igareda, N. (2006). La maternidad en las mujeres presas. Encarceladas-Emakume Atxilotuak XIV Semana Emakume. (pp.60-76). http://www.feministas.org/IMG/pdf/dossier_encarceladas_5_.pdf

Kalinsky, B. (2008). El agente penitenciario: la cárcel como ámbito laboral. Runa, 28(1), 43-57. http://www.pensam ientopenal.com.ar/system/files/2015/01/doctrina37888.pdf

Lamas, M. (2000). Diferencias de sexo, género y diferencia sexual. Cuicuilco, 7(18). https://www.redalyc.org/pdf/35 1/35101807.pdf

Lagarde, M. (1996). “El género”, fragmento literal: 'La perspectiva de género'. Género y feminismo. Desarrollo humano $y$ democracia. España: Ed. homo.

Ley 12.256. Ley de Ejecución Penal Bonaerense. Art 4 (1999). 
Olaeta, H. (2011). Mujeres Privadas de libertad en Argentina: una mirada a partir de los datos provenientes del SNEEP. En Violencia contra las mujeres. Argentina, Ministerio de Justicia y Derechos Humanos de la Nación. Recuperado de: http://www.pensamientopenal.com.ar/system/files/2017/10/doctrina45901.pdf

Pegoraro, J. (2010). Introducción. En P. Calcagno, P. Cucut., L. Grandoso (Comps.) Hablando desde las cárceles. Boletín bimestral publicado por Proyecto Ave Fénix- Facultad de Ciencias Sociales, UBA.

Rodríguez, N. M. (2005). Mujeres madres en prisión en América Central. Costa Rica: Editorial Universidad Estatal a Distancia. Recuperado de https://books.google.co.cr/books?id=jjv2wfhG0CIC\&printsec=copyright\#v=onep age\&q\&f=fals

Yagüe Olmos, C. (2007). Mujeres en prisión. Intervención basada en sus características, necesidades y demandas. Revista Española de Investigación Criminológica, 6 (5). Recuperado de https://dialnet.unirioja.es/servlet/articu lo? codigo $=2477673$

\section{Notas}

1 Marisa Teplisky, licenciada y profesora en sociología (UBA). Especialista en Políticas y Programas Socioeducativos (INFOD- Argentina). Confección de informes técnico-sociales dentro del área de Asistencia social; Coordinadora del Programa de alfabetización en contextos de encierro; Coordinadora de talleres socioeducativos sobre Género y sexualidades (Unidad 40-SPB). Profesora de nivel superior (ISFD N³9 y 113, provincia de Buenos Aires). Lic. Verónica Loyola, licenciada en psicología (UAI). Tratamiento psicoterapéutico y coordinación de dispositivos de asistencia para madres detenidas con hijxs (Unidad 33-Los Hornos, SPB). Elaboración de informes psicológicos forenses. Coordinación de área sociolaboral y talleres sobre género y sexualidades (Unidad 40-Lomas de Zamora, SPB).

2 Datos disponible en.: https://www.argentina.gob.ar/sites/default/files/sneepbuenosaires2015.pdf

3 Datos disponibles en: https://www.argentina.gob.ar/sites/default/files/sneepbuenosaires2017.pdf

4 Los nombres de las participantes han sido modificados para preservar su identidad.

5 La ley $n^{\circ} 27.499$ establece la capacitación obligatoria en género para todas las personas que se desempeñan en la función pública de los tres poderes del Estados.

6 La legislación argentina (ley de Ejecución penal N²4.660) contempla que las mujeres madres pueden vivir con sus hijxs hasta los 4 años de edad y que la infraestructura carcelaria debe estar a adecuada (fundamentalmente debe contar con jardines de infantes). Esto se detalla en el Capítulo XV de la Ley referida. Disponible en: https://www.mpd.gov.ar/up loads/documentos/Ley\%2024660.pdf

7 En las cárceles de la Argentina funcionan escuelas intramuros de nivel primario y secundario las cuales son gestionadas por el Ministerio de Educación (Ley Nacional de Educación $N^{\circ}$ 26206). No obstante, dicho espacio es habilitado para fines socioeducativos, por fuera del horario escolar, cuando se llevan a cabo actividades en el marco del desarrollo estrategias tratamentales, como la del presente taller.

8 "Ni una menos" es una consigna que dio nombre a un movimiento feminista surgido en Argentina en 2015. Es un colectivo de protesta que se opone a la violencia contra la mujer y su consecuencia más grave y visible, el femicidio.

9 Son un conjunto de creencias idealizadas en torno a las formas de vivir y sentir las relaciones amorosas.

10 Las viñetas reflejan estos mitos del amor romántico: "El amor todo lo puede"; "Si te cela es porque te ama", "El mito de la media naranja", "Los opuestos se atraen", "El amor es incondicional".

11 El concepto hace referencia al homicidio de una mujer o persona trans cuando esté motivado por su condición de género.

12 La dinámica del juego fue la siguiente: Lxs participantes se sientan en ronda, alrededor de las tarjetas, que se encuentran distribuidos en el piso con el texto (afirmaciones sobre el campo de la sexualidad y ETS) hacia abajo. Por equipos se levantan de a una y se indica si la información es verdadera o falsa y por qué.

13 Ley 26.485 Ley de protección integral para prevenir, sancionar y erradicar la violencia contra las mujeres en los ámbitos en que desarrollen sus relaciones interpersonales. 